\title{
Ulysse CHEZ KafKa ET Lispector : LE LITTÉRAIRE ENTRE AFFECT ET RAISON
}

\author{
Louis-Thomas Leguerrier
}

Université de Montréal

Bien plus qu'une activité consistant à représenter des idées préconçues ou à consolider des identités individuelles et collectives en les insérant dans un récit historique en compétition avec d'autres récits sur le marché du savoir, la création littéraire permet la confrontation de l'esprit humain avec ce qui le dépasse, ce qui l'appelle tout en lui échappant. Si cette confrontation est mise en scène dans les textes littéraires selon des modalités qui dépendent des conditions historiques de leur production, elle est aussi réactivée, au-delà de ces conditions historiques, chaque fois que l'esprit d'un lecteur, chaque fois que l'esprit d'une lectrice y voit l'occasion d'une ouverture à ce qui l'excède et le fascine.

Un des plus vieux récits de la tradition occidentale illustre de manière frappante cette confrontation de l'esprit avec l'inconnu. Il s'agit de l'Odyssée d'Homère, et notamment du célèbre épisode du chant XII, où Ulysse se ligote au mat de son navire pour pouvoir écouter le chant des Sirènes sans craindre d'y succomber. Grâce à ce stratagème, Ulysse fait l'expérience d'un abandon de l'esprit à ce qui l'affecte de l'extérieur, le fascinant et menaçant de lui faire perdre la raison. Mais en même temps, et dans un même mouvement, il fait l'expérience d'une maîtrise des affects par la force de la raison. À la fois stoïque et ouvert au débordement des affects, Ulysse incarne les extrêmes de l'esprit, le lieu où la tension entre affect et raison est portée à son comble. En prenant l'exemple de deux textes du XXe siècle qui actualisent la figure d'Ulysse, "Le silence des Sirènes » de Kafka et Un apprentissage ou le livre des plaisirs de Clarice Lispector, je voudrais montrer comment les mises en scène du littéraire donnent lieu à une pensée qui n'est épuisée par aucun moment historique particulier ni réductible à aucune discipline institutionnalisée.

Canadian Review of Comparative Literature / Revue Canadienne de Littérature Comparée CRCL DECEMBER 2019 DÉCEMBRE RCLC

0319-051x/19/46.4/421 @ Canadian Comparative Literature Association 


\section{LES SIRÈnES SÉDUITES}

Dans un fragment de 1917, Kafka rejoue la scène de la rencontre entre Ulysse et les Sirènes de manière à produire une nouvelle version de l'histoire :

Comme preuve que des moyens insuffisants, puérils même, peuvent servir au salut : Pour se préserver des Sirènes, Ulysse se boucha les oreilles avec de la cire et se fit enchaîner au mât. Tous les voyageurs, sauf ceux que les Sirènes attiraient de loin, auraient pu depuis longtemps faire de même, mais le monde entier savait que cela ne pouvait être d'aucun secours. La voix des Sirènes perçait tout et la passion des hommes séduits eût fait éclater des choses plus solides que des chaînes et un mât. Mais bien qu'il en eût peut-être entendu parler, Ulysse n'y pensait pas. Il se fiait absolument à sa poignée de cire et à son paquet de chaînes, et tout à la joie innocente que lui procuraient ses petits expédients, il alla audevant des Sirènes. (542)

On sait que dans la version d'Homère, Ulysse ne se bouche pas les oreilles avec de la

cire : il se ligote seulement au mât, afin de pouvoir entendre le chant des Sirènes tout en étant prémuni contre la tentation invincible de se jeter à l'eau pour les rejoindre. Pour sa part, en se bouchant les oreilles avec de la cire, le héros de Kafka semble opter de manière unilatérale pour la fermeture aux affects et pour une maîtrise de soi que ne vient pas provoquer le choc de l'inconnu. Or le texte de Kafka n'est pas aussi simple, puisqu'il indique dès le début que les moyens techniques dont dispose Ulysse sont insuffisants pour le préserver des Sirènes. Ces moyens techniques ainsi que le fait qu'ils doivent si facilement permettre à Ulysse de se fermer à l'expérience extrême que lui réservent les Sirènes sont ridiculisés par Kafka, qui écrit que «le monde entier savait que cela ne pouvait être d'aucun secours » (542). Ulysse se bouchant les oreilles avec de la cire évoque la propension de la raison à se murer dans une citadelle visant à se protéger des affects qui assiègent l'esprit, propension que l'on pourrait illustrer par la figure du stoïcien. Or cette analogie entre Ulysse et le stoïcien se présente seulement sur un plan superficiel, ou encore sous la forme d'une parodie. Le véritable stoïcien n'a pas besoin de se boucher les oreilles avec de la cire ni de quelque autre moyen technique jugé par lui ridicule en face du pouvoir de la raison autonome : il n'a besoin de rien outre la volonté inflexible de rester indifférent face aux choses qui ne dépendent pas de lui. On peut imaginer, quoique cela soit peu probable, qu'un vrai stoïcien, par la seule force de sa raison blindée contre l'expérience sensible, puisse effectivement résister au pouvoir d'attraction surnaturel des Sirènes. Mais même si cela était possible, il aurait quand même perdu. En effet, nous dit Kafka, les Sirènes possèdent une arme secrète : elles peuvent toujours arrêter de chanter :

Or, les Sirènes possèdent une arme plus terrible encore que leur chant, et c'est leur silence. Il est peut-être concevable, quoique cela ne soit pas arrivé, que quelqu'un ait pu échapper à leur chant, mais sûrement pas à leur silence. Au sentiment de les avoir vaincues par sa propre force et à l'orgueil violent qui en résulte, rien de terrestre ne saurait résister. (543)

Tout le sournois des Sirènes est là : écouter leur chant conduit à perdre la raison, mais s'exposer à leur silence conduit à perdre la vie. Si aucun humain ne peut résis- 
ter à la séduction des Sirènes, le sage peut toujours se réfugier dans l'inhumanité de la raison complètement coupée du sensible. Toutefois, en allant aussi loin dans la négation de la vie, il ouvre la porte à l'orgueil monstrueux d'une raison devenue déraison par son délire de puissance, d'une mesure devenue démesure à force de rigueur : « au sentiment de les avoir vaincues par sa propre force et à l'orgueil violent qui en résulte, rien de terrestre ne saurait résister » (Kafka 543). Mais tel ne sera pas ici le sort d'Ulysse, puisque le héros de Kafka n'est qu'une parodie de sage, une mise en scène de l'impuissance et du ridicule dont fait preuve la raison dans son aspiration à l'autonomie et au détachement du sensible. Blanchot, dans Le livre à venir, propose une interprétation du texte de Kafka qui insiste sur l'arrogance d'Ulysse croyant naïvement les Sirènes « vaincues par le pouvoir de la technique qui toujours prétendra jouer sans péril avec les puissances irréelles-inspirées » (11). Or, ce n’est pas la technique rationnelle, ici impuissante, qui permet à l'Ulysse de Kafka d'éviter le péril, mais bien plutôt, précisément, la naïveté avec laquelle il s'en remet au pouvoir supposé de cette technique. C'est cette confiance aveugle d'Ulysse en ses "petits expédients » qui entraîne la confusion en vertu de laquelle Ulysse pourra entendre le silence des Sirènes sans courir aucun danger :

Et de fait, quand Ulysse arriva, les puissantes Sirènes cessèrent de chanter. Soit qu'elles crussent que le silence seul pouvait encore venir à bout d'un pareil adversaire, soit que la vue de la félicité peinte sur le visage d'Ulysse leur fît oublier tous leurs chants. Mais Ulysse, si l'on peut s'exprimer ainsi, n'entendit pas leur silence; il crut qu'elles chantaient et que lui seul était préservé de les entendre ; il vit d'abord distraitement la courbe de leur cou, leur souffle profond, leurs yeux pleins de larmes, leur bouche entrouverte, mais il crut que tout cela faisait partie des airs qui se perdaient autour de lui. Mais bientôt tout glissa devant son regard fixé au loin ; les Sirènes disparurent littéralement devant sa fermeté et c'est précisément lorsqu'il fut le plus près d'elles qu'il ignora leur existence. (Kafka 543)

Face au danger que représente le silence des Sirènes, Ulysse ne perd pas son assurance, puisqu'il attribue le fait qu'il n'entend rien à la réussite de ses expédients, et ne soupçonne pas que c'est uniquement parce que les Sirènes se sont tues volontairement qu'il ne perçoit pas leur chant. La même naïveté qui conduit l'Ulysse de Kafka à se mesurer au chant terrible des Sirènes avec rien d'autre en mains qu'une poignée de cire et quelques chaînes le conduit à se livrer sans peur aucune à leur silence, plus terrible encore. Équipé en apparence pour affronter le chant des Sirènes, c'est à leur silence qu'Ulysse semble finalement le mieux préparé. L'Ulysse de Kafka possède une arme autrement plus puissante que la ruse qui est placée au premier plan dans la version d'Homère : il s'agit de la force d'attraction qui se dégage de sa « joie innocente ", cette "fermeté " avec laquelle il garde son regard " fixé au loin ", rivé à son objectif, la lointaine Ithaque, tellement convaincu que le petit bonheur terrestre qui l'attend là est le seul vrai bonheur qu'il ne peut même pas imaginer être détourné de celui-ci par la séduction des Sirènes, aussi irrésistibles soient-elles. Il y a dans cette attitude comme un stoïcisme inversé qui, les « yeux pleins de larmes » des Sirènes 
en témoignent, est capable d'émouvoir même les monstrueuses créatures qui peuplent la mer aux mille dangers. Kafka propose deux manières d'expliquer pourquoi les Sirènes se sont tues au passage d'Ulysse : « soit qu'elles crussent que le silence seul pouvait encore venir à bout d'un pareil adversaire, soit que la vue de la félicité peinte sur le visage d'Ulysse leur fît oublier tous leurs chants » (543). La première explication conviendrait pour le stoïcien dont la fermeture aux affects est parodiée par la cire dans les oreilles d'Ulysse. Mais pour Ulysse, la seconde est plus plausible, puisque ce qui est mis en scène dans le fragment est la séduction des Sirènes par Ulysse :

Mais elles, plus belles que jamais, s'étirèrent, tournèrent sur elles-mêmes, laissèrent leur terrifiante chevelure flotter librement au vent et leurs griffes se détendirent sur le roc. Elles ne désiraient plus séduire, elles ne voulaient plus que retenir le plus longtemps possible au vol le reflet des grands yeux d'Ulysse. Si les Sirènes avaient eu une conscience, elles se fussent alors anéanties. Mais telles qu'elles étaient, elles restèrent; seul Ulysse leur a échappé. (Kafka 543)

4 Les Sirènes séductrices, pour un instant, sont devenues séduites. Alors que dans l'Odyssée, l'accent est mis sur la manière dont Ulysse se situe par rapport à la séduction des Sirènes, sur sa tendance à se défier de cette séduction tout en y cédant jusqu'à un certain point, ici, l'attention est recentrée sur la situation des Sirènes par rapport à Ulysse, sur la manière dont ce dernier les affecte et les bouleverse. Séduire les Sirènes, ces sombres créatures qui chantent le destin des morts enfermés dans un passé irrévocable, c'est séduire la fatalité de ce passé, c'est infléchir, ne seraitce que pour une seconde, les lois immuables de la nécessité qui limite le possible, puisque, comme l'écrit Kafka, tout le monde sait parfaitement qu'il est impossible pour un humain, quand même il se trouverait dans un cuirassé, de résister au chant des Sirènes. La nécessité (Ananké), est selon les philosophes ce qui contraint toujours et ne peut en aucun cas être persuadé. Faute de quoi, il faudrait admettre l'existence du miracle, et c'est tout l'ordre de la conscience qui serait anéanti. Mais, supposons que, juste une fois, la nécessité se soit laissée persuadée, et cela, à la faveur d'un voyageur qui croyait si fort en ses petits expédients de mortel impuissant que ceux-ci se transformèrent sous ses yeux en attirail de sorcellerie capable d'envoûter les reines de l'envoûtement elles-mêmes, force est de conclure que, " [s]i les Sirènes avaient eu une conscience, elles se fussent alors anéanties » (Kafka 543). Or les Sirènes n’ont pas de conscience, elles ne sont pas soumises, comme l'humain, au principe de noncontradiction, et n'ont pas à se soumettre au dictat de la raison selon lequel le passé est irrévocable. "Seul Ulysse leur a échappé », mais les Sirènes, contrairement au stoïcien qu'elles méprisent et ne daignent même pas gratifier de leurs chants, peuvent très bien souffrir les exceptions. Seul Ulysse a réussi l'impossible : faire que cesse le chant des Sirènes sans avoir à subir le contrecoup de leur mortel silence. Tandis que le sage entend le silence objectif de la vie arbitraire et déraisonnable (les Sirènes) devenue muette face à la rigidité mortifère de la raison guérie du sensible, ce qu'Ulysse entend, pour sa part, est le silence qu'il croit devoir à sa petite tricherie, à ses petits expédients, bref un silence personnel et dépourvu d'objectivité, mais qui trouve sa 
réalité dans le silence extérieur des Sirènes au souffle coupé par le bouleversement intégral qu'elles expérimentent au passage d'un si extraordinaire mortel. Il se peut même, précise Kafka, qu'Ulysse ait été parfaitement conscient de l'effet qu'il fit aux Sirènes, qu'il ait davantage confiance en son redoutable pouvoir de séduction qu'en sa corde et sa cire, et qu'il ne joue la comédie de la naïveté que par ruse et par méfiance, comme il le fait si souvent dans l'Odyssée :

La tradition rapporte d'ailleurs un complément à cette version. Ulysse, dit-on, était si fertile en inventions que la déesse Destinée elle-même ne pouvait lire dans son cœur. Il est possible-encore que l'intelligence humaine ne puisse le concevoir-qu'il ait réellement remarqué que les Sirènes se taisaient et qu'il n'ait usé de la feinte décrite ci-dessus que pour leur opposer, à elles et aux dieux, une espèce de bouclier. (Kafka 543)

Ayant fait mine, comme bien des séducteurs, de ne pas même apercevoir les Sirènes chez qui il venait de provoquer tant d'émoi, Ulysse, suivant cette dernière hypothèse proposée par Kafka, aurait fait la démonstration éclatante qu'il est possible, pour qui en a le courage et l'audace, de retourner la raison contre elle-même et de la contraindre à servir le miracle, l'exception aux lois de la nécessité qu'elle condamne si sévèrement. Quiconque maîtrise la raison s'empressera d'observer qu'il s'agit là d'une hypothèse ridicule. Mais cela ne serait point surprenant, puisque cette hypothèse fait partie des choses que l'intelligence humaine ne peut concevoir. Or Kafka, lui, l'a conçu, et c'est bien toute la force de la pensée littéraire et des figures qui la véhiculent de pouvoir, sans rougir, concevoir l'inconcevable.

\section{Ulysse, Ève ET LE SERPENT}

Dans le roman Un apprentissage ou le livre des plaisirs (1969), Clarice Lispector met en scène la patiente et méticuleuse construction d'une relation amoureuse entre Lori, maîtresse d'école, et Ulysse, professeur de philosophie. Les deux amants, à travers le lent développement de cette relation, traverseront les différentes étapes de ce qui est décrit dans le roman comme un apprentissage de l'amour. L'amour, ici, se présente donc comme quelque chose qui s'apprend, qui est pour ainsi dire l'objet d'un savoir. De nombreuses scènes du roman sont d'ailleurs constituées de discussions entre les deux amants, lors desquelles ceux-ci semblent être à la recherche d'un certain type de connaissance. Or il ne s'agit pas d'un savoir portant sur l'amour, mais plutôt du savoir comme forme d'amour, et de l'amour comme forme de savoir. En apprenant à s'aimer, les deux amants apprennent à s'orienter dans l'univers de la connaissance, et ainsi, développent conjointement une pensée sur les possibilités et les limites de la raison, sur la place de l'être humain au sein de la nature et sur l'articulation de l'intelligible et du sensible au sein de l'esprit.

Ulysse, dans la mise en scène de Lispector, devient donc philosophe professionnel. Or il ne s'agira pas pour lui d'enseigner un savoir philosophique à Lori : 
Au début Lori s'était trompée et avait cru qu'Ulysse voulait lui transmettre certaines choses de ses cours de philosophie, mais il avait dit : «ce n'est pas de philosophie dont tu as besoin, en ce cas ce serait facile : tu assisterais à mes cours comme auditrice et moi je bavarderais avec toi en d'autres termes ». (Un apprentissage 15)

L'apprentissage de Lori concerne un autre domaine, habituellement opposé, bien qu'historiquement entremêlé à celui du savoir philosophique : le domaine de la foi :

D'Ulysse elle avait appris le courage d'avoir la foi-beaucoup de courage, foi en quoi ? Dans sa propre foi, car la foi peut être un grand choc, elle peut signifier tomber dans l'abîme, Lori avait peur de tomber dans l'abîme et serrait une des mains d'Ulysse tandis que l'autre main d'Ulysse la poussait vers l'abîme-bientôt elle devrait lâcher la main moins forte que celle qui la poussait, et tomber. (Un apprentissage 35)

Avoir foi en la foi, bref en ce qui risque de nous précipiter dans l'abîme, c'est là quelque chose qui ne s'apprend pas aisément. Le mouvement qui mène de la raison, qui fonctionne par progression d'un degré à l'autre du savoir, à la foi, qui se situe au-delà ou en

6 deçà de la progression, est un mouvement brusque et imprévisible, un saut, un plongeon, quelque chose comme se jeter tête première en bas de l'échelle. Ce mouvement vers l'abîme, Ulysse apprend à Lori à s'y accoutumer, en l'y poussant d'une main tout en la retenant de l'autre, un peu comme l'Ulysse d'Homère prête l'oreille au chant des Sirènes tout en se retenant par une corde au mât de son bateau. Mais, comme dans le récit de Kafka, les rôles sont inversés par la mise en scène. Ici aussi, c'est Ulysse qui, le premier, séduit les Sirènes. En effet, Lori est associée par Ulysse à la figure des Sirènes séductrices telle que reprise de l'Odyssée par la tradition littéraire allemande :

-C'est dommage que tu t'appelles Lori, parce que Lorelei, c'est ton vrai nom, est plus beau. Tu sais qui était la Lorelei ?

- C'était quelqu'un?

-Lorelei est le nom d'un personnage légendaire du folklore allemand, chanté dans un merveilleux poème de Heine. La légende dit que la Lorelei séduisait les pêcheurs par ses chansons et ils finissaient par mourir au fond de l'eau, je ne me rappelle plus les détails. Non, ne me regarde pas avec ces yeux fautifs. En premier lieu, c'est moi qui te séduis. (Lispector, Un apprentissage 114-15)

Et comme chez Kafka, c'est en renversant la figure du sage, personnification par excellence de la maîtrise des affects par la raison, qu'Ulysse se rend séduisant aux yeux de sa sirène :

À vrai dire, ce qui arrivait à Lori, c'était que, à la suite d'une décision si profonde qu'elle n'en saisissait pas les motifs-elle avait, poussée par la peur, coupé la douleur. Seul Ulysse lui avait permis d'apprendre qu'on ne pouvait pas couper la douleur-sinon on souffrirait tout le temps. Or elle l'avait coupée sans même avoir autre chose qui en soi aurait remplacé la vision des choses par le biais de la douleur d'exister, comme avant. Sans la douleur, elle s'était retrouvée sans rien, perdue dans son propre monde et dans celui des autres sans forme de contact. (Lispector, Un apprentissage 45)

La suppression de l'affect, ici de la douleur, est présentée par Lispector comme un manque, un vide, et de surcroît comme une perte de contact avec le monde, et non 
pas comme la force tranquille, bienheureuse et en communion avec la nature dont se targue le sage aussi indifférent à la douleur qu'à toute forme d'intrusion du monde extérieur au sein du royaume de la raison. Si les Sirènes de l'Odyssée, comme l'ont proposé Adorno et Horkheimer, sont une manifestation de la nature non maitrisée menaçant de faire sombrer l'humain civilisé dans un passé archaïque précédant le développement de la pensée rationnelle, la Lorelei de Lispector, au seuil de sa relation avec Ulysse, est davantage une représentation idéalisée de la nature que la sagesse philosophique plaque par-dessus la nature elle-même afin de la domestiquer : « alors que vous vous exaltez en affectant de lire votre loi dans le livre même de la nature, vous visez en fait le contraire [...]. Votre orgueil entend régenter jusqu'à la nature et lui inculquer votre morale et votre idéal " (Nietzsche 27), reprochait Nietzsche jadis aux stoïciens. En un siècle où a été éliminé jusqu'au souvenir d'une nature qui n’aurait pas été ainsi dressée par l'idéal, les Sirènes elles-mêmes ont appris la discipline qui consiste à se couper de toute douleur, et seul Ulysse, peut-être, est encore capable de les arracher à leur mortel détachement.

Tel est donc l'enjeu de l'apprentissage qui est au cœur de la liaison entre Ulysse et Lori : trouver un moyen d'être en harmonie avec la nature sans transformer celle-ci en une pure projection de l'idéal derrière laquelle disparait la véritable nature. Et cet enseignement, c'est en vertu de la forme unique d'intelligence qui est la sienne, lui, le professeur de philosophie qui ne veut pas parler de philosophie, qu'il est en mesure de le prodiguer :

Elle seule paraissait vouloir apprendre de lui quelque chose et elle s'était trompée en pensant qu'elle voulait apprendre du fait qu'Ulysse était professeur de philosophie, et qu'elle se servirait de lui dans cet espoir. Une fois perdu celui-ci, voyant qu'il n'avait pas la moindre intention de lui enseigner un mode de vie "philosophique » ou « littéraire », il était déjà trop tard : elle était attachée à lui parce qu'elle voulait être désirée. (Lispector, Un apprentissage 46)

Alors que Lori attendait de la part d'Ulysse un savoir philosophique, un savoir qui ferait d'elle une projection idéalisée de la raison, c'est en vertu d'une toute autre chose qu'elle se trouve séduite et qu'elle ressent le besoin d'être désirée par lui. Cette autre chose, on l'a vu, pourrait être appelée la foi : « D'Ulysse elle avait appris le courage d'avoir la foi » (Lispector, Un apprentissage 35). Mais cela demande à être explicité. La mise en scène de Lispector, comme d'habitude, est loin d'être simple. En effet, si Ulysse est celui qui enseigne la philosophie, c'est quand même Lori, et non celui-ci, qui se trouve du côté du savoir, du moins au début de l'apprentissage. L'incipit du roman est à cet égard éclairant :

tellement occupée, à cause des courses que la bonne avait faites à la va-vite, car de plus en plus elle bâclait son travail, même si elle ne venait que pour préparer le déjeuner et le dîner, elle avait donné plusieurs coups de téléphone pour régler des problèmes, le plus difficile pour appeler le plombier, elle était allée à la cuisine ranger les achats et disposer dans la corbeille les pommes qui étaient sa meilleure nourriture [...], alors du ventre même, comme une secousse lointaine de terre dont on sait à peine que c'est un séisme, 
de l'utérus, du cœur contracté vient le tremblement gigantesque d'une violente douleur ébranlée, de tout le corps l'ébranlement-en subtiles grimaces du visage et du corps, avec la difficulté d'un pétrole déchirant la terre-vient finalement la grande crise de larmes sèches, une crise muette sans aucun son qu'elle put même entendre, qu'elle n'avait pas prévue-secouée comme l'arbre solide qui est plus profondément ébranlé que l'arbre fragile-finalement canaux et veines éclatés. (Lispector, Un apprentissage 11-12)

Cette scène d'ouverture lors de laquelle Lori a ses règles évoque sans le dire une des plus anciennes mises en scène du problème du savoir, c'est-à-dire le récit biblique du péché originel, dans lequel Ève, la première femme, est séduite par le serpent qui la conduit à goûter au fruit défendu de l'arbre de la connaissance du bien et du mal, figure par excellence de l'autonomie du savoir humain. Dans cette première scène du roman, la mention de la pomme comme "meilleure nourriture " suivie de celle du châtiment de Dieu : "je multiplierai tes souffrances et tes grossesses : c'est dans la souffrance que tu enfanteras des fils » (Ancien Testament 11), permettent de supposer que Lori, qui se dit d'ailleurs « ornée toute comme une femme biblique " (Lispector, Un apprentissage 17), lorsqu'elle commence son apprentissage, possède déjà le savoir qu'elle a pensé un moment obtenir d'Ulysse. Cela permet aussi de supposer que ce savoir, fruit de l'arbre de science, en même temps qu'il découvre à l'être humain de nouvelles possibilités, le prive de ce qu'aucune sagesse ne pourra lui redonner, c'est-àdire l'arbre de vie. C'est vers cette vie située par-delà bien et mal que l'apprentissage d'Ulysse et Lori les achemine progressivement. Alors que le roman s'ouvre sur une évocation du péché originel, tout le développement de celui-ci décrit le chemin du retour à l'innocence. Mais comment, à partir de la vie domestiquée par le savoir, la vie idéalisée et par le fait même déchue, s'élever à une vie située au-delà du savoir ? Pour qui veut affronter un tel problème, la forme particulière d'intelligence qui est celle d'Ulysse apporte un secours inespéré. N'est-ce pas parce qu'il ignore qu'aucune corde ni aucune cire ne constituent une véritable protection contre le chant des Sirènes, bref en vertu de son non-savoir, qu'Ulysse, dans le récit de Kafka, parvient à si bien s'en tirer? Et ce non-savoir, loin de la simple ignorance, n'est-il pas en fait une autre forme d'intelligence ? C'est ce que laisse penser la mise en scène de Kafka. De même chez Lispector, dont le récit précise qu'Ulysse, si l'apprentissage qu'il propose à Lori n'est pas proprement philosophique, est tout de même professeur de philosophie. C'està-dire qu'il connaît la raison et sait en faire usage, mais qu'il choisit de s'attacher à une autre forme d'intelligence qui, si elle ne sert en rien la sagesse philosophique, lui ouvre les portes d'une vie où le miracle est encore possible.

Avoir foi en cette vie, malgré le fait que l'on sache parfaitement que la raison la condamne et la disqualifie, voilà l'épreuve que Lori voudrait avoir le courage de supporter, courage qu'elle espère conquérir auprès d'Ulysse. Comme ce dernier l'explique dans un long monologue, si ce qui se gagne par le non-savoir est si difficile à obtenir, c'est parce que quiconque tente d'y accéder se met à avoir honte en face du savoir, comme quand Adam et Ève, juste après avoir goûté au fruit défendu, se mirent à avoir honte de leur nudité : 
Mais regarde tous ceux qui t'entourent et vois ce que nous avons fait de nous et ce que nous considérons comme notre victoire de chaque jour [...]. Nous n'avons pas accepté ce qu'on ne comprend pas, parce que nous ne voulons pas passer pour des imbéciles [...]. Nous avons essayé de nous sauver, mais sans utiliser le mot salut pour ne pas avoir honte d'être innocents. [...] Nous n'avons pas été purs et ingénus pour éviter de nous moquer de nous-mêmes et à la fin du jour pouvoir dire « au moins je n'ai pas été idiot » et ainsi ne pas être perplexes avant d'éteindre la lumière. Nous avons souri en public de ce dont nous ne sourions pas quand nous sommes seuls. Nous avons appelé faiblesse notre naïveté. Nous nous sommes craints les uns les autres, par-dessus tout. Et c'est tout cela que nous considérons comme notre victoire de chaque jour. Mais moi j'y ai échappé, Lori, j'y ai échappé avec la férocité qui permet d'échapper à la peste, et j'attendrai que toi à ton tour tu sois davantage prête. (Lispector, Un apprentissage 54-56)

Qui possède déjà le savoir, aurait-il l'intuition qu'une vie plus vraie et plus ample existe et qu'il suffit pour y accéder de faire un seul pas du côté du non-savoir, risque fort de ne jamais faire ce pas, puisque certaines choses, comme le fait de ne pas passer pour un idiot, valent plus aux yeux du savoir que la vie elle-même quelle que soit son ampleur. C'est ainsi que Lori, à travers sa relation avec Ulysse, trouve une formulation pour des vérités que le savoir a expulsées dans le domaine des choses qu'on ne peut pas dire, sous peine de dire n'importe quoi et de passer pour des idiots. Prenons par exemple cette affirmation qui, si elle peut paraître plutôt inoffensive, représente un véritable scandale pour le savoir :

« Ne pas comprendre » était si vaste qu'il dépassait un quelconque comprendrecomprendre était toujours limité. Mais ne-pas-comprendre n'avait pas de frontières et conduisait à l'infini, à Dieu. Ce n'était pas un ne-pas-comprendre comme pour un simple d'esprit. Le bon c'était avoir une intelligence et ne pas comprendre. C'était une bénédiction étrange comme celle d'être atteinte de démence sans être folle. C'était un désintérêt tranquille par rapport aux choses dites de l'intellect, une douceur de stupidité [...]. Comprendre était toujours une erreur-elle préférait l'espace si ample et libre et sans erreur qu'était le ne-pas-comprendre. (Lispector, Un apprentissage 49)

Affront direct à la maxime de Spinoza selon laquelle il ne faut pas tourner en dérision les affaires humaines, ni les prendre en pitié ou en haine, mais les comprendre, l'attirance de Lori pour le « ne pas comprendre » et son idée selon laquelle ce dernier serait plus vaste, plus libre et moins erroné que le "comprendre » semble tout à fait adaptée à la forme d'intelligence d'Ulysse qui, tel qu'on l'a vu chez Kafka, ne parvient à se sortir de situations impossibles que parce qu'il comprend mal les situations en question. Or le « ne pas comprendre " de Lori est aussi en accord, lorsqu'on le prend par un autre côté, avec la pensée de Spinoza. Dès son premier roman, Lispector mentionne explicitement Spinoza (Près du cour sauvage 176-77). Et par la suite, elle abordera constamment des thèmes liés à la pensée de celui-ci dans le cadre de sa production romanesque. Cependant, ni Un apprentissage ni aucun de ses autres romans ne correspondent à ce qu'on pourrait être tenté d'appeler une représentation littéraire des idées de Spinoza. Tout comme la figure d'Ulysse telle qu'elle apparaît chez Kafka, chez Lispector et chez les autres qui seront abordés bientôt, n'est ni un personnage 
conceptuel ni la représentation littéraire d'une quelconque pensée philosophique, mais plutôt une expérience de pensée visant à concevoir ce que la raison juge inconcevable, l'œuvre de Lispector, dans ses aspects spinozistes, est une tentative de concevoir par le langage une partie du système de Spinoza qui échappe complètement au domaine de la représentation. Divisé en trois genres de connaissance, l'édifice spinoziste comporte une partie sensible (le premier genre), une partie rationnelle (le deuxième genre) et une partie intuitive (le troisième genre). Le passage entre le premier et le deuxième genre de connaissance engage une confrontation entre l'esprit et ce qui le dépasse qui est plutôt fidèle au modèle du stoïcien : affecté par les représentations internes émanant de l'expérience sensible, le philosophe, dit Spinoza, doit prendre acte du bouleversement intérieur avec lequel il est aux prises, et ensuite soumettre ces représentations au jugement de la raison qui pourra en valider certaines et en invalider d'autres. Mais Lispector, elle, s'intéresse plutôt à l'articulation entre le deuxième et le troisième genre de connaissance, la connaissance rationnelle et la

10 connaissance intuitive. La question qui traverse son œuvre en général et Un apprentissage en particulier est la suivante : comment, à partir de la raison qui comprend, faire le saut vers un «ne pas comprendre » qui offre une connaissance beaucoup plus ample de la réalité, une connaissance directe et immédiate, qui ne soit pas limitée par la médiation du comprendre. Selon Spinoza, l'accès à ce genre de connaissance est réservé au philosophe qui a su traverser les affects donnés dans la connaissance sensible pour ensuite les dépasser dans la connaissance rationnelle et finalement retrouver, dans la connaissance intuitive, une joie et une béatitude incomparablement plus complètes que les plaisirs éphémères de l'expérience sensible, une joie qui est celle de l'union parfaite avec Dieu, c'est-à-dire le Tout de la nature. Accéder à la connaissance intuitive, pour Spinoza, revient à accéder au point de vue de Dieu, et le Dieu de Spinoza n'a pas besoin de la médiation du comprendre, il est ce que Lispector appelle un " ne pas comprendre " plus ample, plus libre et plus parfait que le comprendre. En effet, c'est un Dieu semblable à celui de Spinoza dont Lori recherche la proximité :

Le vrai Dieu, qui n'était pas fait à son image et à sa ressemblance, pour cette raison lui était totalement incompréhensible, et elle ne savait pas s'Il pourrait la comprendre. Son Dieu jusqu'à présent avait été terrestre, et rien de plus. Désormais si elle voulait prier, ce serait comme prier à l'aveuglette le cosmos et le Néant. Elle découvrit que jusqu'à alors elle avait prié pour un moi-même, certes puissant, grandi et omnipotent, qu'elle appelait le Dieu, et tel qu'un enfant voit son père comme la figure d'un roi. Ensuite Lori s'éveilla un peu à une réalité plus objective autour d'elle, elle changea la position de sa tête sur bras plié. Elle se dit que quelques minutes auparavant elle luttait avec le Dieu, fatiguée, épuisée, elle murmura sans timbre de voix : je ne comprends rien. C'était une vérité si indubitable que son corps tout comme son âme se ployèrent légèrement et ainsi elle put se reposer un peu. En cet instant elle n'était qu'une des femmes du monde, et non un moi, et elle s'intégrait en quelque sorte à une marche, éternelle et sans objectifs, d'hommes et de femmes en pèlerinage au Néant. Ce qui était le Néant était exactement le Tout. (Lispector, Un apprentissage 76-77) 
Se détournant du Dieu humain, trop humain, de la religion chrétienne-un Dieu à notre image auquel on s'adresse comme à un père ou à un roi et qui, comme nous, se trouve séparé de la nature-pour partir à la recherche d'un Dieu qui correspond au Tout de la nature et dans lequel la différence entre nature humaine et nature non humaine s'efface, Lori tente d'effectuer le saut du deuxième au troisième genre de connaissance. Pour comprendre, il faut être un humain séparé de la nature non humaine, et faute de pouvoir maîtriser complètement cette nature, le sage plaquera sur elle une projection idéalisée de celle-ci. Or pour s'unir vraiment au Tout de la nature sans l'idéaliser, il faut cesser de comprendre, cesser de passer par la médiation du savoir, et accéder à l'immédiateté de la connaissance intuitive. Si Spinoza pose cette connaissance comme l'aboutissement d'une démarche proprement philosophique et réserve la béatitude qu'elle procure au philosophe, Lispector montre bien qu'une telle connaissance a quelque chose d'un au-delà de la philosophie elle-même, et elle fait de cette étrangeté fondamentale du passage entre le deuxième et le troisième genre de connaissance le moteur d'une expérience de pensée littéraire qui, elle, vise résolument à se déployer par-delà les limites de la pensée philosophique. Lispector propose donc beaucoup plus qu'une répétition de Spinoza : elle arrache la pensée spinoziste à son contexte strictement philosophique et y mêle des impuretés tirées de la tradition biblique. Si le savoir comme péché originel correspond à l'état initial de Lori dans le roman, état dans lequel elle tente, suivant le modèle de la raison retranchée dans sa citadelle imprenable, de couper complètement la douleur que provoque le contact avec le monde, alors le « ne pas comprendre » ainsi que la foi qu'il faut avoir pour s'y maintenir malgré la peur de passer pour des idiots correspond à un retour à l'innocence qui précède le péché originel. Ce retour à l'innocence est perceptible dans une scène située en plein milieu du roman, lors de laquelle Lori prend un bain de mer au petit matin :

La mer était là, la plus incompréhensible des existences non humaines. Et là était la femme, debout, le plus incompréhensible des êtres vivants [...]. Elle et la mer. [...] Il devait être six heures du matin. Le chien en liberté hésitait sur la plage, le chien noir. Pourquoi est-ce qu'un chien est si libre ? Parce qu'il est un mystère vivant qu'on ne cherche pas à éclaircir. La femme hésite parce qu'elle va entrer. Son corps se console de sa propre exiguïté par rapport à l'immensité de la mer parce que c'est l'exiguïté de son corps qui permet à celui-ci de devenir chaud et délimité, et ce qui faisait d'elle une personne pauvre et libre, avec sa part de liberté de chien sur le sable. Ce corps entrera dans le froid illimité qui sans rage rugit dans le silence du petit matin. La femme ne le sait pas : mais elle accomplit un acte de courage. Avec la plage vide à cette heure, elle n'a pas l'exemple des autres humains qui transforment l'entrée dans la mer en simple jeu futile de vivre. La mer salée n'est pas seule parce qu'elle est salée et grande et c'est là une réalisation de la Nature. Le courage de Lori c'est, ne se connaissant pas, d'avancer malgré tout, et agir sans se connaître exige du courage. (Lispector, Un apprentissage 91-92)

Cette rencontre entre Lori, représentante de la nature humaine, et la mer qui personnifie ici la nature non humaine par excellence a lieu dans une ambiance de création et de commencement. En effet, cette femme qui n'a pas encore l'exemple des autres 
humains pourrait très bien être la première femme. La mention de l'heure, six heures du matin, rappelle le sixième jour de la création dans la Genèse, lors duquel Dieu créa l'être humain, et le fait que la femme doive faire preuve de courage pour avancer malgré qu'elle ne se connaisse pas rappelle la situation qui précède le péché originel, c'est-à-dire une situation dans laquelle la pomme n’a pas encore été mangée, dans laquelle le savoir autonome, par l'entremise du fruit de l'arbre de la connaissance du bien et du mal, n'a pas encore été découvert par l'être humain. À ce moment précis de l'histoire humaine, la femme se trouve devant un choix : ou bien elle mange la pomme et s'empare du comprendre, ce qui la coupera de la nature non humaine qui deviendra pour elle une projection idéalisée, ou bien elle se maintient dans l'innocence $\mathrm{du}$ « ne pas comprendre » et ainsi, s'unit avec la mer de manière à s'intégrer au Tout incompréhensible de la nature. Lori, pour sa part, choisit la seconde option :

En avançant, elle fend en deux les eaux du monde. Elle n'a plus besoin de courage, désormais elle est ancienne comme le rituel retrouvé qu'elle avait abandonné des millénaires auparavant [...]. Les mains en conque et avec la superbe de ceux qui ne donneront jamais d'explications, pas même à eux-mêmes-dans la conque des mains pleine d'eau, elle boit à grandes gorgées, bonne pour la santé d'un corps. Et c'était ce qui lui manquait : la mer en elle comme le liquide épais d'un homme [...]. Ensuite elle marche dans l'eau pour revenir à la plage, et les vagues la poussent doucement pour l'aider à sortir. Elle ne marche pas sur les eaux-ah, elle ne ferait jamais cela du moment que des millénaires auparavant on avait déjà marché sur les eaux-, mais personne ne lui enlèvera cela : marcher dans les eaux. (Lispector, Un apprentissage 93-94)

S’étant accouplée avec la mer de manière à se perdre un instant dans le Tout indivisible de la nature libérée des limites que lui impose la volonté de comprendre, Lori retrouve l'innocence abandonnée des millénaires auparavant, lorsque les premiers humains goûtèrent au fruit défendu. Mais contrairement au Christ qui transcende la nature et se trouve au-dessus de celle-ci, elle ne marche pas sur les eaux, mais dans les eaux, et ainsi, elle joint l'immanence du Dieu-nature de Spinoza à l'univers de références bibliques qu'elle met par ailleurs en place. Le rôle joué dans la scène par la mer évoque aussi, bien sûr, le personnage d'Ulysse, dont l'absence dans cette scène pourrait bien s'expliquer par le fait que c'est Lori elle-même qui, ici, assume le rôle du héros homérique : "D’une certaine façon obscure, ses cheveux défaits sont ceux d'un naufragé. Car elle sait-elle sait qu'elle a provoqué un danger. Un danger aussi ancien que l'être humain » (Lispector 94). À propos de Un apprentissage, Nadia Batella Gotlib écrit :

Les voyages sur un bateau qui affronte la mer en y plongeant déterminent l'axe commun des images du roman, fondées sur un rapport entre le moi et l'autre. Donc, quoique ce soit un discours centré sur la femme, il se réalise dans le rapport de la femme avec l'autre qui est elle-même, qui est l'homme, qui est Ulisses, qui est le Monde. (71)

En effet, la séduction ne peut pas ici être réduite au rapport hétérosexuel présupposé par le fait que Lori est une femme et Ulysse un homme, mais concerne plus largement la tension jamais résolue au sein de l'esprit entre affect et raison. Que cette tension se 
manifeste à l'intérieur d'une seule personne, entre une personne et le monde extérieur, ou encore entre deux amoureux, dans ce contexte, devient secondaire. Se laisser séduire est toujours chose dangereuse, que le séducteur soit le serpent de la Genèse ou l'Ulysse de Lispector, puisqu'il s'agit toujours de se laisser entraîner par l'affect qui instaure sans que l'on puisse le contrôler un certain rapport entre l'humain et la nature, un certain ordre de la connaissance. C'est en suivant un affect qu'Ėve, séduite par le serpent, «le plus rusé de tous les animaux » (Ancien Testament 9), goûte au fruit interdit à la source de la raison autonome qui se retournera ensuite contre l'affect, jugé trop arbitraire, en se coupant de la nature non humaine. De même, c'est en suivant l'affect provoqué par sa rencontre avec Ulysse, le plus rusé de tous les mortels, que Lori instaure un nouvel ordre de la connaissance qui réunit l'humain et la nature non humaine au sein du Dieu immanent.

Chez Kafka comme chez Lispector, la figure d'Ulysse déborde manifestement le cadre de l'Odyssée pour se déployer en tant que mouvement d'une pensée reposant sur une constellation d'éléments -la raison, l'affect, et leur conflit au sein de l'esprit - pouvant être mis en scène d'une infinité de manière différente. En réactivant la mise en scène homérique et en l'utilisant pour aborder le rapport de l'esprit à ce qui l'affecte et le dépasse, Kafka et Lispector attestent d'une pensée mise en marche et véhiculée par les œuvres littéraires, une pensée qui transcende autant le contexte historique de leur production que leur insertion institutionnelle au sein d'une discipline ou d'une tradition.

\section{Ouvrages CITÉS}

Blanchot, Maurice. Le livre à venir. Gallimard, 2005.

Dhorme, Edouard, éditeur. La Bible. 1: L’Ancien Testament. Gallimard, 2000.

Kafka, Franz. Euvres complètes. 2. Édité par Claude David, traduit par Marthes Robert, Gallimard, 1997.

Gotlib, Batella. «Un apprentissage des sens ». Études françaises, vol. 25, no. 1, pp. 69-80, doi.org/10.7202/035773ar

Lispector, Clarice. Un Apprentissage ou le livre des plaisirs. Traduit par Jacques et Teresa Thiérot, Des femmes, 1992.

---. Près du coeur sauvage. Traduit par Regina Helena, Des femmes, 1981.

Nietzsche, Friedrich. Par-delà bien et mal. Traduit par Cornelius Heim, Gallimard, 1971.

Spinoza, Baruch. Traité politique. Traduit par Charles Appuhn, Garnier Flammarion, 1964, pp. 12-13. 